\title{
Multipoint Measurement Technique for Tracking Electromagnetic Interference Propagation and Correlation in a Complex Installation
}

\author{
Muhammad Imam Sudrajat ${ }^{1,2}$, Niek Moonen ${ }^{1}$, Hans Bergsma ${ }^{3}$, Rob Bijman ${ }^{3}$, Frank Leferink ${ }^{1,3}$ \\ ${ }^{1}$ University of Twente \\ Enschede, \\ The Netherlands
${ }^{2}$ Indonesian Institute of Sciences -
LIPI, \\ Indonesia
${ }^{3}$ THALES Nederland B.V., Hengelo,
The Netherlands

\begin{abstract}
Electromagnetic interference (EMI) can degrade the power quality $(P Q)$ in an electrical network. Identification of the interference source and its propagation can only be achieved by distributed measurement methods using synchronous multipoint measurements. This paper proposes a multipoint measurement analysis method using the number of coincidence events and a coincidence ratio to determine the relationship between each EMI event at several measurement locations. The measurement is performed using six distributed power quality analyzers.
\end{abstract}

Keywords-Electromagnetic interference, power quality, multipoint measurement, number of coincidence, coincidence ratio

\section{INTRODUCTION}

Power distribution in an electrical network is not only concerned with supplying power but also related to maintaining PQ. The PQ issue is defined as the deviation of the voltage and current characteristics from sinusoidal, symmetrical and nominal conditions [1], [2]. PQ degradation in the electrical network is not only caused by the quality of the generator but also related to the characteristic of the installed load. Next to modern equipment being more vulnerable to power supply deviations, it is also causing a lot of EMI in the $2 \mathrm{kHz}-150 \mathrm{kHz}$ bandwidth [3]-[5]. Typical conducted EMI events are harmonic distortion, flicker, voltage surge, burst, voltage dip, voltage sag, voltage interruption, rapid voltage change (RVC) and swell. These do not only cause issues for the system that is directly connected to the interference source, but it also propagates and interferes with other systems or subsystems that are located further away.

The common view towards $P Q$ is that it is a supply problem; i.e. interference due to a PQ issue is caused by the mains supply, and interference from equipment is causing a distortion of the mains. In islanded systems, the power supply is not from the mains but produced by, for instance, a diesel generator, which is a weaker configuration compared to a mains connection. Interference caused by some equipment will propagate in a complex installation and interfere other equipment. Information Technology Industry Council (ITIC) has published an AC voltage boundary curve [3]. This curve has been used as a reference to establish a PQ performance measure.

In a power grid with a floating ground or isolated terra (IT) system, such as on shipboard, PQ problems can also cause

\footnotetext{
This project has received funding from the European Union's Horizon 2020 research and innovation programme under the Marie Skłodowska-Curie grant agreement No 812391.
}

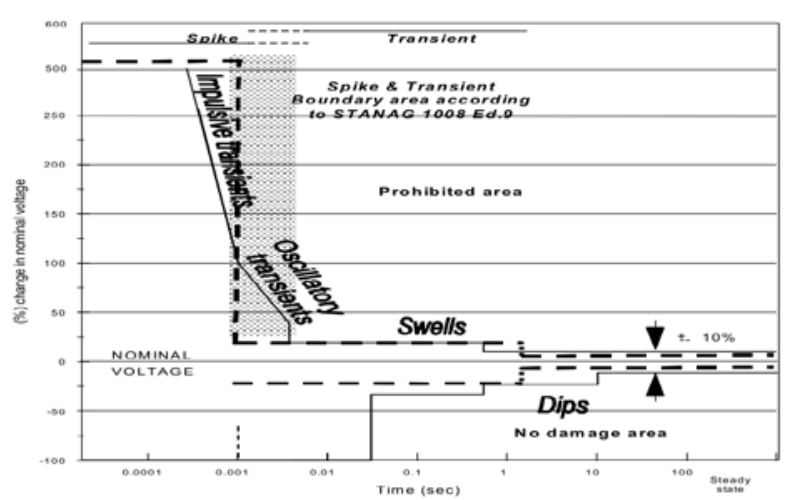

Fig. 1. Voltage limit curve comparison: STANAG 1008 (dash) and ITIC (solid) [5].

malfunctions or even damages to equipment and systems, as it is an islanded power system. Characteristic of PQ shipboard electrical system is referring to STANAG 1008 ed.9. Fig. 1 shows a comparison between the ITIC and STANAG 1008 curves. A distributed measurement system is needed to determine the impact of EMI in complex installations like an islanded power grid. A wide variety of distributed measurement methods have been proposed to assess the cause of voltage and current distortions in the general power distribution system [1], [6], [7]. Also, more local multipoint measurement systems have been recently developed [8], [9]; however, these are limited to a range of several meters and require post-processing of many TBytes to detect PQ related EMI events. Compared with previous research, this paper provides a technique that can record EMI events specifically, which often require high sampling rates. Through synchronous recording, the correlation between each EMI event at different locations can be determined.

This paper describes a simultaneous multipoint measurement system. Correlation between EMI events happening throughout a complex installation onboard a ship is investigated through the coincidence of events using a Venn intersection approach. This method has been applied in a real electrical distribution network in a ship to find the root cause of a malfunctioning device. First, in Section II the measurement setup as implemented in the electrical distribution system is described, followed by the analysis method of the acquired data in Section III. In Section IV the results are presented, followed by the discussion and conclusion in Sections V and VI, respectively.

\section{MEASUREMENT SETUP}

The measurement system consists of six power quality analyzers model PQube 3 manufactured by PSL-Power 
Standard Lab, which acquires 512 samples per $50 \mathrm{~Hz} / 60 \mathrm{~Hz}$ cycle [10]. They are interconnected through a router, while a Raspberry pi-3 is used for time reference. Each PQube is equipped with 8 GByte memory to store the data. Fig. 2 shows the measurement system configuration, as was implemented in the distribution network. The data obtained is the result of event recording data from the six PQubes which performed measurements in accordance with IEC 61000-4-30 and were programmed using a higher level to measure voltage deviations based on STANAG-1008. Table I show the measurement threshold setting. The data was collected over 21 days during normal journey operation of the ship.

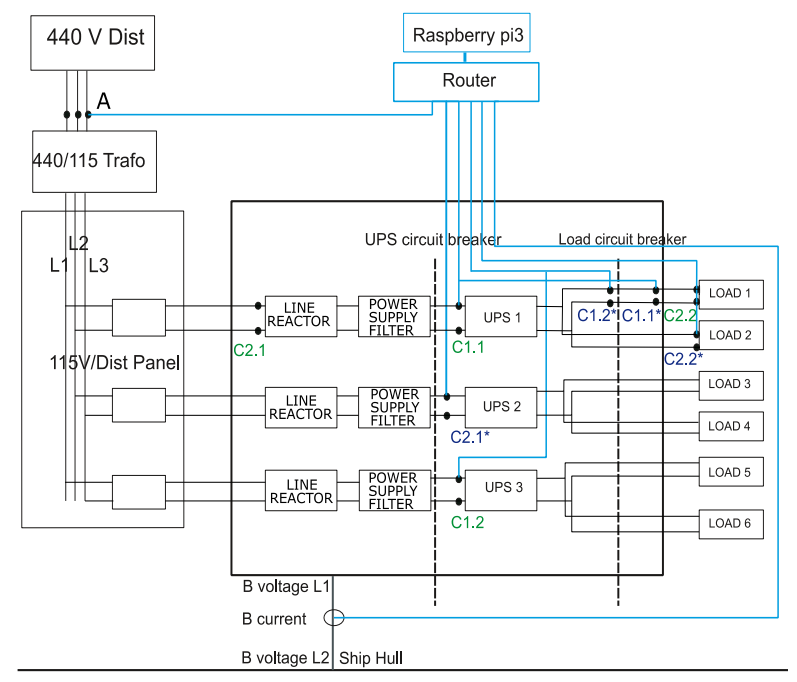

Fig. 2. Measurement points of PQubes.

TABLE I. MEASUREMENT THRESHOLD SETTING COMPARED WITH STANAG 1008 ED.9 [11].

\begin{tabular}{|l|l|l|}
\hline Parameters & \multicolumn{1}{|c|}{ STANAG 1008 ed.9 } & \multicolumn{1}{|c|}{$\begin{array}{c}\text { Measurement threshold } \\
\text { setting }\end{array}$} \\
\hline Dip & $-16 \%(2 \mathrm{~s})$ & $\begin{array}{l}-6 \%(2 \mathrm{~s}) \\
-10 \%(1 \mathrm{~s}) \\
-14 \%(0.05 \mathrm{~s})\end{array}$ \\
& & $-5 \%(<$ dip duration) \\
\hline Sag & - & $>90 \%$ \\
\hline Interruption & - & $-5 \mathrm{up} \mathrm{to} \mathrm{+5 \%}$ \\
\hline RVC & - & $200 \mathrm{~V}($ except PQ A, \\
\hline Impulse $/$ & $2.5 \mathrm{kV}, 440 \mathrm{~V}$ & $1000 \mathrm{~V})$ \\
spike & $1 \mathrm{kV}, 115 \mathrm{~V}$ & \\
\hline Swell & $0.6 \mathrm{kV}, 24 \mathrm{Vdc}$ & $+5 \%$ \\
\hline
\end{tabular}

The stored data is analyzed by recreating a timeline based on the folder structure of each PQube. The folder names contain information about the specific event and at which time it occurred. In total, 31 GB of data was gathered in 21 days. The measurement was set up to specifically record dip, sag, interruption, RVC, impulse, and swell events in accordance with IEC 61000-4-30. So data is not continuously stored every day, but only the relevant data that triggered by abnormalities in the voltage and current.

\section{ANALYSIS METHOD}

As the amount of data was huge, a formal analysis approach was developed. Two methods for analyzing the results are explored in detail. The first method is merely counting the number of events occurring in specific PQube that coincides with events in other PQubes. This is called the number of coincidence and can be mathematically expressed as:

$$
n_{\text {coincidence }}=\Sigma\left(P Q_{1 \text {,event } \mathrm{x}} \cap P Q_{2, \text { event } \mathrm{y}}\right)
$$

With PQx being A, B, C1.1 to C2.2. While the event can be a dip, sag, interruption, RVC, impulse or swell. From this, one can directly see which events are occurring most often.

The second method is the coincidence ratio. The coincidence ratio is calculated using the Venn intersection compared with the total sum (union) approach. An intersection is an event that occurs simultaneously on $\mathrm{PQ}_{1}$ with another specific event occurring in any of the other PQubes. This data is then compared with the total sum of the two specific events in the two PQubes. The equation used to calculate this coincidence ratio $(\beta)$ is:

$$
\beta=\frac{\Sigma\left(\mathrm{PQ}_{1, \text { event } \mathrm{x}} \cap P Q_{2, \text { event } \mathrm{y}}\right)}{\Sigma\left(P Q_{1, \text { event } \mathrm{x}} \cup P Q_{2, \text { event } \mathrm{y}}\right)}
$$

\section{ANALYSIS RESUlT}

The results are presented in the number of coincidence and the coincidence ratio. Both use a dataset that was collected in 21 days, except for PQube B. Events recorded by PQube B can only be shown during the last 11 days because of the limitations of SD card memory. The recorded data, with the events as the function of time, is presented in Fig. 3.

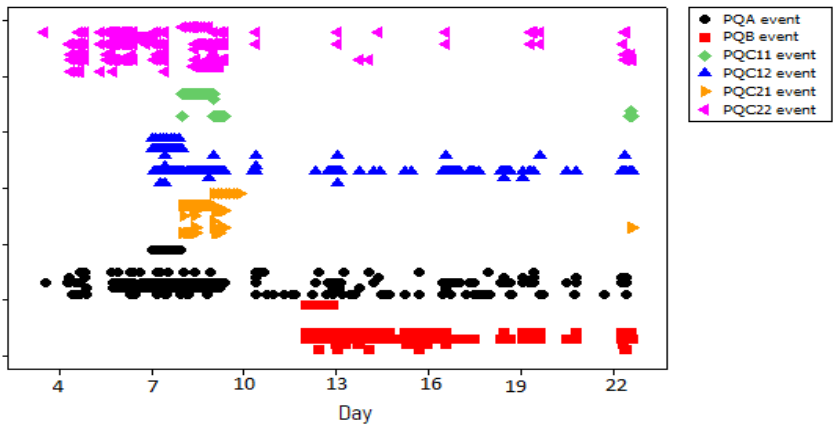

Fig. 3. Recorded electromagnetic interference event history.

For both analysis methods, the same two cases are examined in detail as test cases for the proposed approach. The first case is taken from the coincidental event, which is related to voltage interruption in the load recorded by PQube C2.2 (C22 int). The interruption event is represented the load's circuit breaker switch off or trip. The second study case is taken from the coincidental events which are related to an RVC event recorded by PQube A ( $\left.\mathrm{A}_{\mathrm{RVC}}\right)$.

\section{A. Number of coincidence events method}

The number of coincident events is calculated by applying a 6 seconds window, which means events occurring 6 seconds before and 6 seconds after the event in C2.2 are considered. This is related to the nature of the event in C2.2, which is 1 to 6 seconds earlier than other PQubes events. Equation (1) is used to calculate the result. The total number of coinciding events recorded by each measurement point for 21 days of observation that are related to C22 ${ }_{\text {int }}$ are shown in Table II. In the second case, the number of coincidence events recorded by each PQube related to $A_{R V C}$ is shown in Table III. 
TABLE II. NUMBER OF OtHER PQ RECORDED EVENT COINCIDE WITH THE OCCURRENCE OF INTERRUPTION AT C2.2 (C22 $\left.2_{\mathrm{INT}}\right)$

\begin{tabular}{|l|c|c|c|c|c|c|}
\hline Event & A & C22 & C11 & C21 & C12 & B \\
\hline Dip & 0 & - & 1 & 6 & 4 & 0 \\
\hline Sag & 53 & - & 5 & 4 & 13 & 18 \\
\hline Interruption & 0 & - & 0 & 0 & 0 & 0 \\
\hline RVC & 6 & - & 0 & 0 & 0 & 0 \\
\hline Impulse & 0 & - & 0 & 0 & 0 & 0 \\
\hline Swell & 1 & - & 0 & 0 & 0 & 8 \\
\hline
\end{tabular}

TABLE III. Number of OTHER PQ RECORdED EVENT COINCIDE WITH THE OCCURRENCE OF RVC AT A ( $\left.\mathrm{A}_{\text {RVC }}\right)$

\begin{tabular}{|l|c|c|c|c|c|c|}
\hline Event & A & C22 & C11 & C21 & C12 & B \\
\hline Dip & - & 4 & 0 & 1 & 0 & 0 \\
\hline Sag & - & 1 & 57 & 0 & 132 & 25 \\
\hline Interruption & - & 6 & 0 & 0 & 0 & 0 \\
\hline RVC & - & 0 & 1 & 56 & 0 & 0 \\
\hline Impulse & - & 0 & 0 & 0 & 0 & 0 \\
\hline Swell & - & 0 & 0 & 0 & 0 & 17 \\
\hline
\end{tabular}

\section{B. Coincidence ratios method}

In this subsection, the results are presented in a similar fashion as the previous subsection, however now (2) is used to calculate the coincidence ratios. Again the results are presented related to $\mathrm{C} 22_{\text {int }}$ in the first case and $\mathrm{A}_{\mathrm{RVC}}$ in the second case. The results are shown in Table IV and Table V, respectively.

TABLE IV. EVENT CORRELATION AT EACH PQUBE WITH C2.2 INTERRUPTION EVENTS (C22 $2_{\text {INT }}$ COINCIDECE RATIOS \%)

\begin{tabular}{|l|c|r|r|r|r|c|}
\hline Event & A & C22 & C11 & C21 & C12 & B \\
\hline Dip & $0,0 \%$ & - & $\mathbf{1 2 , 5 \%}$ & $\mathbf{1 0 , 2} \%$ & $\mathbf{1 0 , 0 \%}$ & $0,0 \%$ \\
\hline Sag & $\mathbf{8 , 0 \%}$ & - & $3,0 \%$ & $\mathbf{1 4 , 3 \%}$ & $2,0 \%$ & $0,2 \%$ \\
\hline Interruption & $0,0 \%$ & - & $0,0 \%$ & $0,0 \%$ & $0,0 \%$ & $0,0 \%$ \\
\hline RVC & $2,1 \%$ & - & $0,0 \%$ & $0,0 \%$ & $0,0 \%$ & $0,0 \%$ \\
\hline Impulse & $0,0 \%$ & - & $0,0 \%$ & $0,0 \%$ & $0,0 \%$ & $0,0 \%$ \\
\hline Swell & $1,0 \%$ & - & $0,0 \%$ & $0,0 \%$ & $0,0 \%$ & $1,3 \%$ \\
\hline
\end{tabular}

Table V. Event Correlation at Each PQube With $\mathrm{A}_{\text {rvc }}$ Events $\left(\mathrm{A}_{\mathrm{RVC}}\right.$ COINCIDENCE RATIOS \%)

\begin{tabular}{|l|c|c|c|c|c|c|}
\hline Event & A & C22 & C11 & C21 & C12 & B \\
\hline Dip & - & $2,1 \%$ & $0,0 \%$ & $1,3 \%$ & $0,0 \%$ & $0,0 \%$ \\
\hline Sag & - & $0,5 \%$ & $\mathbf{7 1 , 3} \%$ & $0,0 \%$ & $\mathbf{8 8 , 0 \%}$ & $0,2 \%$ \\
\hline Interruption & - & $3,1 \%$ & $0,0 \%$ & $0,0 \%$ & $0,0 \%$ & $0,0 \%$ \\
\hline RVC & - & $0,0 \%$ & $1,3 \%$ & $\mathbf{7 0 , 0 \%}$ & $0,0 \%$ & $0,0 \%$ \\
\hline Impulse & - & $0,0 \%$ & $0,0 \%$ & $0,0 \%$ & $0,0 \%$ & $0,0 \%$ \\
\hline Swell & - & $0,0 \%$ & $0,0 \%$ & $0,0 \%$ & $0,0 \%$ & $2,8 \%$ \\
\hline
\end{tabular}

\section{DISCUSSION}

The used multipoint measurement technique used in this paper has recorded many EMI events occurring onboard a ship. This measurement technique combined with the number of coincidence analysis method and/or the coincidence ratio analysis method. The number of coincidence method is suitable to calculate the number of occurrences and the coincidence ratio method is suitable to calculate how strong the correlation of each event with other events. In the case of the interruption event at the load (C22 int), from Table II it followed that mainly sag events at different locations were occurring. However, from the coincidence ratio presented in Table IV, one can see it didn't have much effect on other subsystems as their ratios are around $10 \%$. The 53 events recorded at position A, give an overestimation of correlation when compared to the coincidence ratio of $8 \%$. This is related to the total number of sag events happening at $\mathrm{A}$, as the probability to occur simultaneously will increase drastically while it might be independent for the interruption at C22. From the second case, both the number of coincidence and coincidence ratio method shows a similar result. It shows that when an RVC is happening at $\mathrm{A}$ it has the potential to interfere with the performance of other subsystems. Table V shows the highest coincidence ratio for $\mathrm{C} 12_{\text {sag }} 88.0 \%$ (132 events), $\mathrm{C} 11_{\text {sag }} 71,3 \%$ (57 events), and C21 $1_{\text {sag }} 70.0 \%$ (56 events).

\section{CONCLUSION}

A multipoint measurement method with six distributed PQubes has been developed to investigate the root cause of (conducted) EMI on a complex installation. The multipoint measurement method can be used to detect the propagation of EMI in any direction within a distribution network. The threshold level for events of the PQubes was set very tight, resulting in a massive amount of data. Two formal analysis methods have been used to investigate the correlation between EMI events recorded by six PQubes. The first method showed the number of coinciding events, i.e. it showed how many events occurred with the same timing related to the initial event. This can, however, be an overestimated figure depicting correlation as the events counted could have been occurring independently of the initial event under investigation. The second method takes this into account and provides a coincidence ratio that shows how much relevance an event has when occurring during the event under investigation. These formal analysis methods have shown that interruption events in the load are related to voltage dips at nearby subsystems and sags occurring in the power supply systems; however, causality has yet to be investigated.

\section{ACKNOWLEDGEMENT}

The authors wish to thank J. Baardink, I. Purwahyudi, D. Kurniawan, for their help performing the measurement.

\section{REFERENCES}

[1] C. Muscas, L. Peretto, S. Sulis, and R. Tinarelli, "Investigation on multipoint measurement techniques for PQ monitoring," IEEE Trans. Instrum. Meas., vol. 55, no. 5, pp. 1684-1690, 2006.

[2] IEC Std 61000-4-30:2015, "Electromagnetic Compatibility (EMC) - Part 4-30: Testing and measurement techniques - Power quality measurement methods.” 2015.

[3] F. Leferink, "Conducted interference, challenges and interference cases,” IEEE Electromagn. Compat. Mag., vol. 4, no. 1, pp. 78-85, 2015.

[4] "CENELEC TR 205A Investigation results on electromagnetic interference in the frequency range below $150 \mathrm{kHz}$, CLC/FprTR 50669,” CENELEC, 2017.

[5] J. Prousalidis, E. Styvaktakis, E. Sofras, I. K. Hatzilau, and D. Muthumuni, "Voltage dips in ship systems," IEEE Electr. Sh. Technol. Symp. ESTS 2007, no. September 2014, pp. 309-314, 2007.

[6] L. Cristaldi, A. Ferrero, and S. Salicone, "A distributed system for electric power quality measurement,” IEEE Trans. Instrum. Meas., vol. 51, no. 4, pp. 776-781, 2002.

[7] K. V. Suslov, N. N. Solonina, and A. S. Smirnov, "Distributed power quality monitoring,” Proc. Int. Conf. Harmon. Qual. Power, ICHQP, 2014.

[8] T. Hartman, N. Moonen, and F. Leferink, "Evaluation of Multichannel Synchronous Conducted TDEMI Measurements for High Voltage Power Electronics," in Proc. of the 2018 International Symposium on Electromagnetic Compatibility (EMC Europe 2018), 2018, vol. 2018Augus, pp. 839-843.

[9] T. Hartman, N. Moonen, and F. Leferink, "Direct sampling in multichannel synchronous TDEMI measurements,” in 2018 IEEE 4th Global Electromagnetic Compatibility Conference (GEMCCON), 2018.

[10] Power Standards Lab, PQube 3 Power Analyzer Specification. 2019.

[11] STANAG 1008, "Characteristics of shipboard electrical power systems in warships of the NATO navies - edition 9.” NATO, 2004. 
